\title{
New Routes to the Preparation of Silver-Doped Sol-Gel Films for a SERS Study
}

\author{
Jae Soo Kang, Chul Jae Lee, Mak Soon Kim, and Mu Sang Lee \\ Department of Chemistry Education, Kuthgpook Kational University, Daegu 702-701, Korea \\ Received April 15, 2003
}

\begin{abstract}
New methods were developed to prepare silver-doped sol-gel films for surface-enhanced Raman spectroscopy (SERS) applications. First. silver ions were doped into a sol-gel matrix. The doped silver ions were reduced into corresponding silver metal particles by two reductive procedures: chemical reduction and thermal reduction. The SERS spectra of benzoic acid were used to demonstrate the SERS effect of the new substrates. The adsorption strength of benzoic acid adsorbed on differently reduced substrates was discussed. The possible adsorption form and the orientation of adsorbate were also discussed.
\end{abstract}

Key Words : Silver-doped sol-gel film. Surface-enhanced Raman spectroscopy. SERS-active substrate. Benzoic acid

\section{Introduction}

Although Raman spectroscopy has been used considerably as an analytical tool for quite some time, the small scattering cross-section inherent with the Raman technique and its possibly high background fluorescence are disadvantages of Raman spectroscopy. The discovery in the 1970s of as much as a 10 -fold enthancement in the Raman signal for compounds adsorbed on roughened electrodes has renewed considerable interest in the field of Raman spectroscopy. Two theories exist explaining the origins of this enhancement; the short-range chemical model and the long-range electronagnetic model. The theory and spectrometric data of SERS have been extensively reviewed in the literature. ${ }^{2}$ Although numerous reports have been published with the mechanisnis of SERS. some analytical studies employing SERS have also appeared in the literature. ${ }^{3}$ In general. the observation of the SERS effect requires the presence of nanometer roughness $(10-100 \mathrm{~nm})$ on the metal surface. The previous analytical applications employed metal-coated microspheres. ${ }^{4}$ silver island films. ${ }^{5}$ LB film. ${ }^{6}$ and colloids ${ }^{7,8}$ as the SERS-active substrates. Recently, a special issue on Raman scattering for the sol-gel process has been published. It is reported that porous silicates derived from the solgel process are excellent support for Au particles and provide a new way for studying SERS. ${ }^{11}$

In this paper, easy-to-prepare and cost-effective substrate for obtaining SERS enthancement is presented. The new substrate consists of silver-doped sol-gel filnus deposited onto a solid support such as a glass microscope slide. In order to evaluate the possibility as SERS-active substrate. we observed the SERS spectra of benzoic acid adsorbed on the silver-doped sol-gel filmis.

\section{Experimental Section}

Instrumentation. Raman and SERS measurements were conducted with a SPEX $14030.85 \mathrm{~m}$ double spectrometer equipped with a gallium-arsenide photonultiplier tube (RCA
C31034). Data storage and processing were handled with DM3000R software and 486 personal computer. The 514.5 num line of an argon-ion laser (INNOVA 90-5. Coherent) was used for excitation. Unless othenvise indicated. the laser excitation power was set at $100 \mathrm{~mW}$. A $90^{\circ}$ angle geometry of the laser excitation source with respect to the collecting optics was used. The monochromator slit widths were set at $600 \mu \mathrm{m}$. The sensitivity of the spectrophotometric system was routinely calibrated with a capillary tube filling with pure $\mathrm{CCl}_{4}$ or a $\mathrm{TiO}_{2}$ disk. FT-Raman spectrum was obtained with a BRUKER FRA 106/S system. Photomicrograph was obtained with a Hitachi $\$-4200$ scanning electron microscope (SEM).

Chemicals. Tetramethyl orthosilicate (TMOS, Aldrich), methanol (CARLO ERBA), silver nitrate (JUNSEI), benzoic acid (BA. ЛUNSEI). sodium benzoate (SB. ЛNSEI), sodium borohydride (Aldrich). and nitric acid (JUNSEI) were analytical reagents or equivalent and used without further purification. Triply distilled water was used for preparing the sample solutions and as solvent for BA.

Procedure. Silver nitrate salt was chemically or thermally reduced in thin xerogel films prepared from TMOS. Glass microscope slides (thickness $\sim 1 \mathrm{~mm}$. Coming) were cut into $10 \times 25 \mathrm{~mm}$ sections, washed in detergent. rinsed in deionized water. soaked in $2 \mathrm{M}$ nitric acid for $2 \mathrm{lh}$, rinsed again and stored in methanol until use. The preparation of the substrate was as follows: working at room temperature, 0.5 $\mathrm{M}$ silver nitrate solution containing nitric acid was prepared. In a small vial. TMOS. methanol, and silver nitrate solution were mixed in a volume ratio of $1: 2: 1$. When these solutions were mixed and stirred, they began to react; the hydrolysis and condensation reactions continued to take place while the mixture aged. Thus, there exists a particular ageing time-window for which the sol-gel solution is coatable. Shortly after the mixing of the solutions, a suspension of colloidal particles (sol) is produced due to the initial hydrolysis and polycondensation reactions between TMOS and water. Sometime after the mixing of the solutions, the time-window is usually bounded by the gelation time, at 
which the interconnection between these particles increases; the viscosity of the sol starts to increase, which eventually leads to the formation of a solid gel. When a sol-gel preparation is in a viscous liquid stage (sol), it may be conveniently coated on a solid support in order to form uniform thin films. Under the present experimental conditions, the time-window between liquid sol and solid gel was about 45 -days when the vial cap was closed. We, therefore, had enough time to handle the sol-gel solutions.

The silver-doped sol-gel films were prepared by a dipcoating process consisting of three steps: dipping. withdrawal and heating. After $48 \mathrm{~h}$ ageing time, the pre-cleaned glass plate was dipped into the homogeneous TMOSmethanol-silver nitrate solution for $3 \mathrm{~s}$. Immediately after the glass plate was withdrawn from the solutions using the tweezers, the glass plate was placed even with the ground. Then the sol-gel solutions spreaded onto the glass plate, adhered to the glass surface, and solidified slowly through evaporation of the solvent. The glass plate coated with solgel film was left to dry in air at an ambient temperature for $30 \mathrm{~min}$ and then baked in an oven at $90{ }^{\circ} \mathrm{C}$ for $12 \mathrm{~h}$. This heating process removes the excess water and solvent within the sol-gel matrix.

The silver ions trapped within the xerogel films were reduced to silver metal particles by chemical and thermal reduction. The chemical reduction was as follows: the glass plate was soaked in $0.02 \mathrm{M}$ sodium borohydride solution. After a few seconds, the glass plate was rinsed thoroughly with water and methanol to remove any excess sodium borohydride. The thermal reduction was as follows: the glass plate was heated in an electric furnace at 300 " $\mathrm{C}$ for $1 \mathrm{~h}$. For later convenience, we labelled the chemically reduced substrate as CRS and the thermally reduced substrate as TRS.

Before measurement, substrates were cut into further $10 \times$ $15 \mathrm{~mm}$ sections. To make use of the prepared substrate, a 20 $\mu \mathrm{l}$. of $10^{-3} \mathrm{M}$ BA solution was pipetted onto it. After the evaporation of the solvent water, the substrate was ready for SERS analysis.

\section{Results and Discussion}

It has been shown by several researchers that surface roughness plays a critical role in the observation of the SERS effect. ${ }^{11,12}$ The Ag-doped xerogel film was characterized using a scanning electron microscope (SEM). Figure l shows the SEM photograph of TRS, which gave an optimum SERS enhancement. The SEM photograph of the substrate demonstrates that the silver nanoparticles are almost uniform in size, with an estimated average of $50 \mathrm{~nm}$ in diameter.

In order to observe the structural changes of TMOS during the ageing time, we obtained the normal Raman spectra of reagents that are composed of the sol-gel solution. From Figure 2, we could see that the TMOS is hydrolyzed in a solgel solution. All the spectra showing in the same figure have the same intensity scale. Figure $2(a)$ is the normal Raman

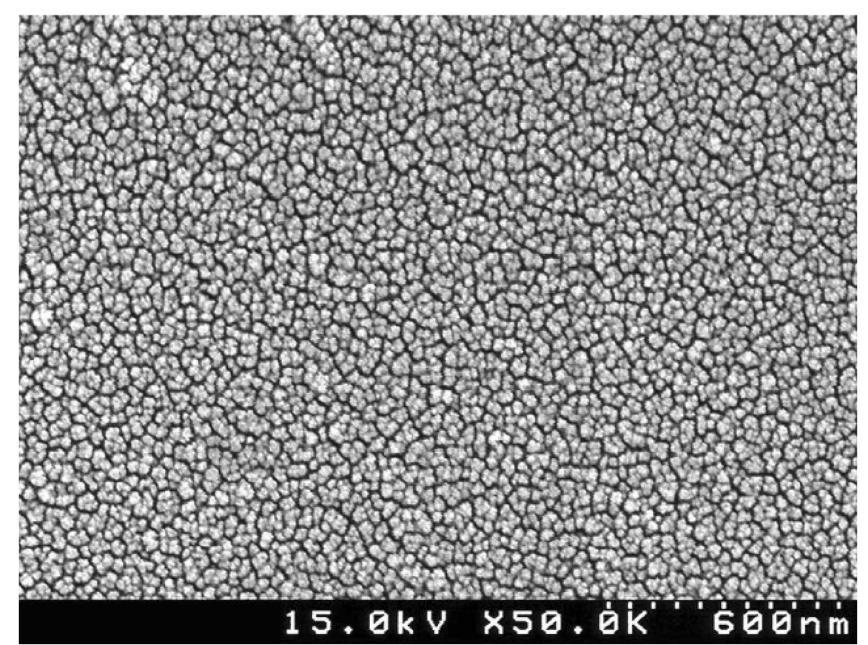

Figure 1. SLM micrograph of a thermally reduced xerogel film (TRS).

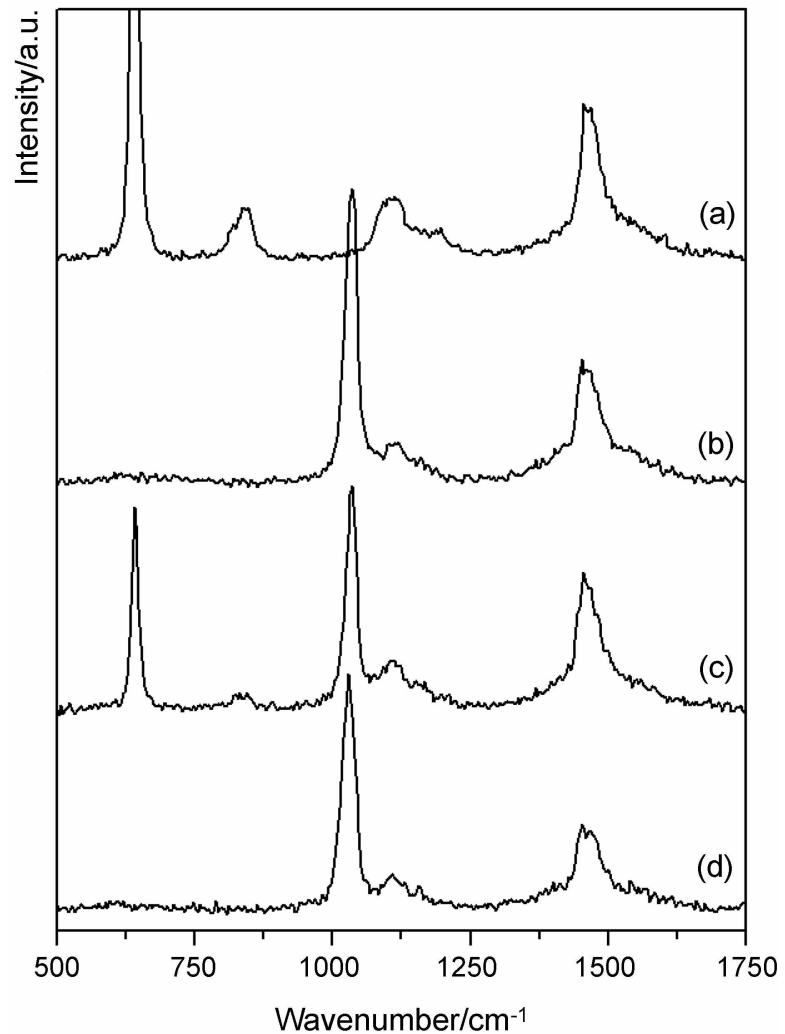

Figure 2. Vormal Raman spectra of (a) TMOS, (b) McOH. (c) TMOS and MeOH mixtures, and (d) sol-gel solution.

spectrum of pure [MOS. The peak at $656 \mathrm{~cm}^{-1}$ is assigned to the Si-O-C symmetric stretching, which shows very strong intensity because of a highly symmetrical structure of IMOS. Figure 2(c) is the normal Raman spectrum of the IMOS and methanol solutions which are mixed in a volume ratio of $1: 2$. In Figure $2(\mathrm{c})$, we still can observe a peak at $656 \mathrm{~cm}^{-1}$ which proves the retention of a symmetrical structure of TMOS. Only the peak intensity is decreased because the concentration of IMOS in the mixture is diluted. Figure 2(d) is the normal Raman spectrum of TMOS. 


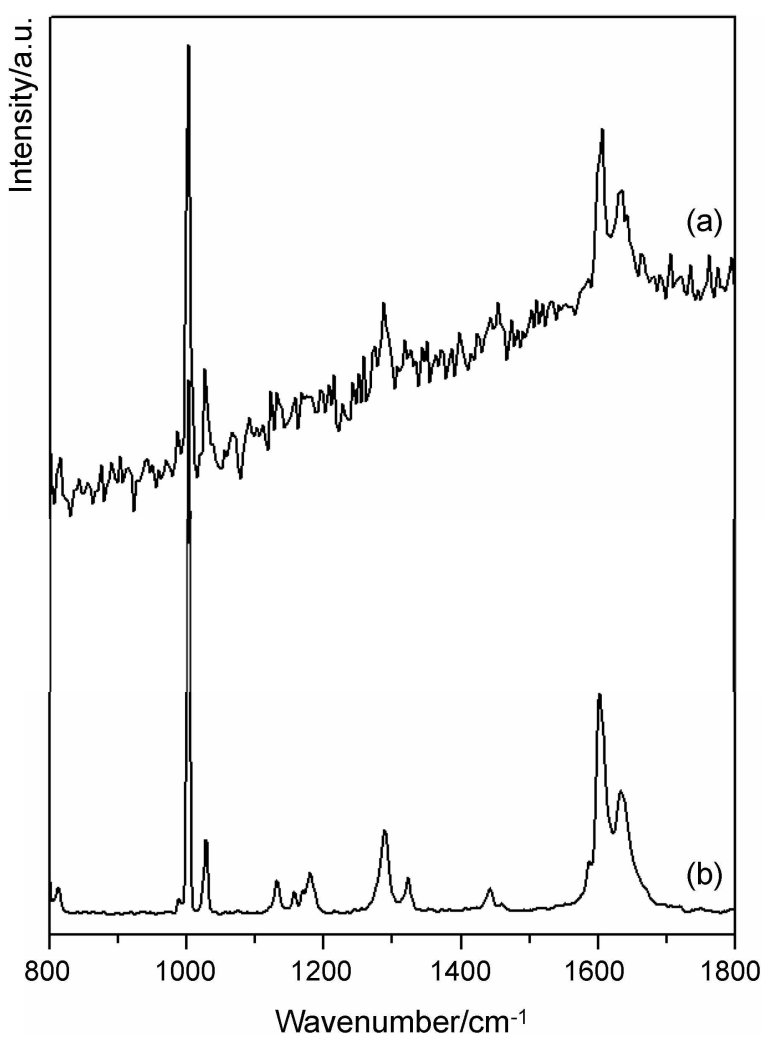

ligure 3. Spectra of pure $B \Lambda$ in a solid state. (a) Vormal Raman and (b) l"I-Raman.

methanol, and $0.5 \mathrm{M}$ silver nitrate solutions which are mixed in a volume ratio of $1: 2: 1$, that is the same composition as explained in the experimental part. In Figure $2(\mathrm{~d})$, the peak at $656 \mathrm{~cm}^{-1}$, which was observed in Figure $2(a)$ and $2(\mathrm{c})$, is no longer observed. 'This means that the highly symmetrical structure of TMOS is destroyed resulting from hydrolysis between the TMOS and water. We have confirmed from Figure 2 that the hydrolysis reaction proceeds first, and then an equilibrium state is retained between a hydrolysis and a condensation step. [his is because an acidic catalysis tends to increase the rate of hydrolysis and disfavor condensation reactions, whereas a base hydrolysis produces rapid condensation. In the initial reaction stage, the yield of product by condensation reactions is low. Moreover, the Raman active mode of the siloxane ( $\mathrm{Si}-\mathrm{O}-\mathrm{Si}$ ) bond vibration is weak. So in Figure 2(d). we are unable to observe the Raman peak related product by condensation reactions.

The normal Raman spectrum and the F"l-Raman spectrum of pure BA in a solid state are measured to compare with the SERS spectrum obtained using the substrate prepared in the present study and are shown in Figure 3. For the same purpose, the normal Raman spectra of pure $\mathrm{SB}$ are measured and are shown in Figure 4. We will also utilize figure 3 and 4 to explain the possible adsorption form and the orientation of adsorbed BA. The normal Raman spectrum of pure BA in a solid state shows a background fluorescence when an argon ion laser of $514.5 \mathrm{~nm}$ line is used for the excitation source. The Fl-Raman spectrum is measured again to display a Raman signal that can not be identified certainly by

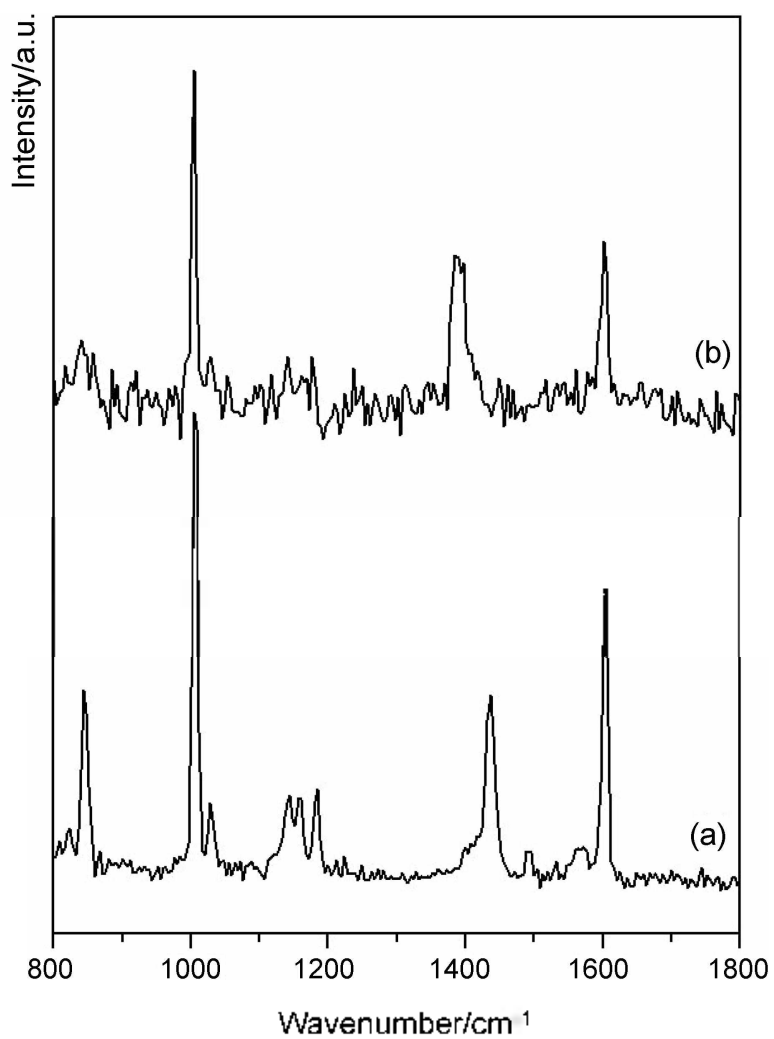

Figure 4. Normal Raman spectra of pure $S 13$ in a (a) solid state and (b) $0.5 \mathrm{M}$ acjucous solution.

background fluorescence. According to previous reports, ${ }^{13-15.18}$ $\mathrm{BA}$ is adsorbed in benzoate form when it adsorbs on the SERS substrate surface. Figure 4(b) offers the normal Raman spectrum of benzoate form, which has an equal form in an aqueous solution state of BA that it is impossible to

Table 1. Vibrational frecquencies ( $\mathrm{cm}^{1}$ ) and assignments of BA and SB

\begin{tabular}{|c|c|c|c|c|c|}
\hline \multicolumn{3}{|c|}{$13 \mathrm{~A}$} & \multicolumn{2}{|c|}{$\mathrm{Sl} 3$} & \multirow[t]{2}{*}{ Assignnา } \\
\hline $\mathrm{ORS}^{a}$ & FTRS $^{h}$ & SERS & Solid & Solution" & \\
\hline 420 & 420 & & & & $16 a$ \\
\hline 618 & 618 & & & & $6 \mathrm{~b}$ \\
\hline \multirow[t]{2}{*}{794} & 795 & & & & 11 \\
\hline & & 840 & 844 & 840 & $\delta(C O O)$ \\
\hline 1002 & 1001 & 1008 & 1004 & 1004 & 12 \\
\hline \multirow[t]{3}{*}{1026} & 1026 & 1028 & 1028 & & $18 \mathrm{a}$ \\
\hline & 1132 & $1] 44$ & & & $9 \mathrm{~b}$ \\
\hline & & & 1184 & & 9 \\
\hline \multirow[t]{4}{*}{1286} & 1287 & & & & $V(C-())$ \\
\hline & & 1392 & & 1392 & $v_{\mathrm{s}}\left(\mathrm{COO}^{-}\right)$ \\
\hline & & & 1436 & & $19 \mathrm{~b}$ \\
\hline & & & 1492 & & $19 \mathrm{a}$ \\
\hline 1606 & 1603 & 1604 & & & $8 \mathrm{a}$ \\
\hline 1634 & 1634 & & & & $v(\mathrm{C}=\mathrm{O})$ \\
\hline 3070 & 3071 & 3064 & & & $v(\mathrm{C}-\mathrm{H})$ \\
\hline
\end{tabular}

"Ordinary Raman spectrum. "FT-Raman spectrum. 'Denoted in terms of Wilson notation given in Vassanyi. (i. Assignments for Tibrational Spectrt of Seven Hundred Benzene Derrictives: Academia Kindo: Budapest. 1974. 
measure by a solubility problem (while I $\mathrm{g}$ of $\mathrm{SB}$ dissolves to $1.8 \mathrm{~mL}$ of water at room temperature, but only $3.4 \mathrm{~g}$ of BA equals $1 \mathrm{~L}$ ). The peak assignments of $\mathrm{BA}$ and $\mathrm{SB}$ are done with reference to literature ${ }^{1.3}$ and are given in Table 1.

Before measuring the SERS spectra of $\mathrm{BA}$, we investigated the spectra of substrates only. No SERS enhancement due to the sol-gel film or its additive was observable in the present investigation.

Shown in Figure 5 are the SERS spectra of $10^{-3} \mathrm{M} \mathrm{BA}$ obtained using Ag-doped sol-gel films and show several bands which correspond to the BA bands reported previously. ${ }^{14}$ Were there not surface enhancements, one would have failed to observe the Raman bands of a diluted solution such as $10^{-3} \mathrm{M}$. The SERS spectrum of BA obtained using CRS [Figure 5(a)] is very similar with T. Vo-Dinh ef al's result that was obtained using an Ag-coated alumina substrate. ${ }^{15}$ The broad bands between 1200 and $1600 \mathrm{~cm}^{-1}$ from Figure 5(a) are most likely due to the Raman scattering from a graphitic carbon layer created by the laser decomposition of a BA monolayer. ${ }^{16.17}$ When the SГRS spectrum of $\mathrm{BA}$ is obtained using TRS [Figure 5(b)], however, these broad bands are decreased critically and, in addition, the SERS bands of $\mathrm{BA}$ are more enhanced.

Why cannot we observe the broad bands when the SERS spectrum of BA is obtained using TRS? In order to answer this question, an experimental measurement was conducted to find the variation of the SERS spectrum of BA with laser illumination power. BA can be photolyzed especially at a higher laser excitation power. Figure 6(a) is the SERS

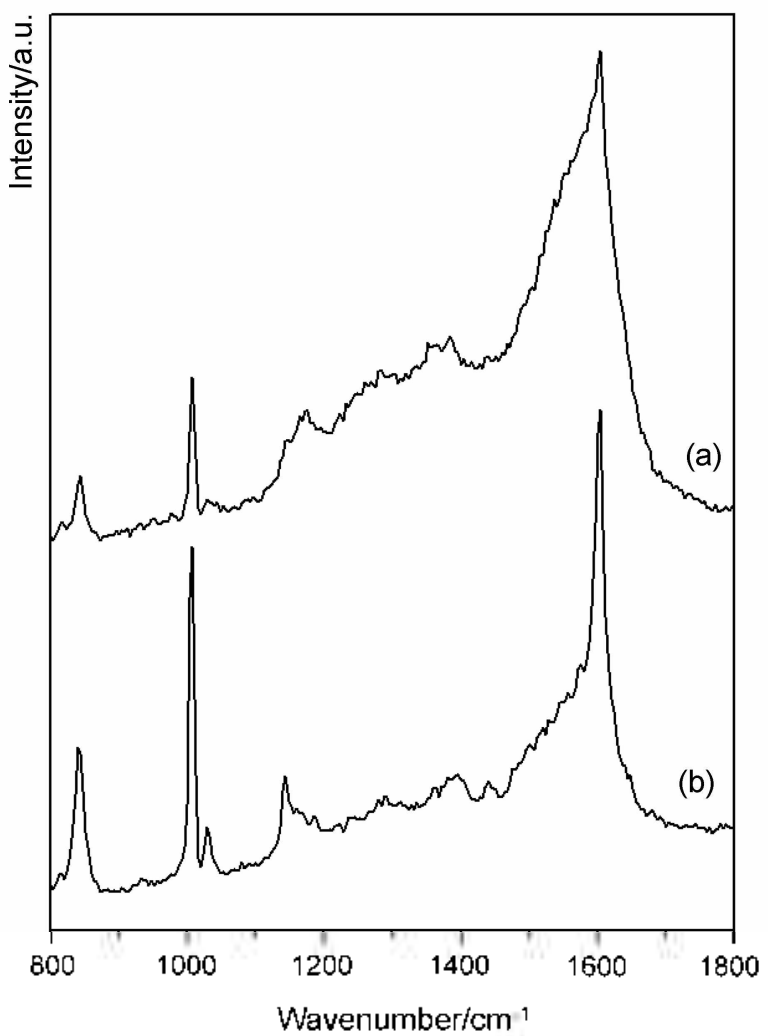

Figure 5. SLRS spectra of $10^{-5} \mathrm{M} B A$ adsorbed on (a) CRS and (b) TRS. spectrum of BA obtained using TRS that is measured with $100 \mathrm{~mW}$ laser power for the first time, which is the same spectrum shown in Fìgure 5(b). After measuring Figure 6(a). the laser excitation power is increased to $200 \mathrm{~mW}$ and the sample. which is used for measuring the Figure 6(a), is illuminated with a laser for $10 \mathrm{~min}$. Then, the laser excitation power is adjusted to $100 \mathrm{~mW}$ again and the sample is rescanned for a second time, which is shown in Figure $6(b)$. Despite the illumination of the sample with a higher laser excitation power for $10 \mathrm{~min}$, we are unable to observe Raman scattering from a graphitic carbon in Figure $6($ b) as can be seen in Figure 5(a). Only the overall SERS enhancement is reduced.

From the results of Figure 5 and Figure 6 , we have made the following conclusion; the adsorption strength of $\mathrm{BA}$ on Ag-doped sol-gel films is different according to the kind of reduction method. BA adsorbs on CRS strongly, whereas it adsorbs on TRS weakly. When BA adsorbs on CRS strongly, it decomposes to graphitic carbon rather than desorbs from the surface by the energy of a laser. In case of the adsorption on TRS, BA desorbs from the surface by the increased energy of a laser because it adsorbs on surface weakly. So the overall SERS intensity is reduced because of the reduced $\mathrm{BA}$ concentration when the spectrum is measured for a second time.

Pagannone ef al. ${ }^{1 \cdot 1}$ and Kwon et al. ${ }^{18}$ have reported the SERS study of BA. The former group suggested that the BA molecules adsorb on the silver particles through the carbox-

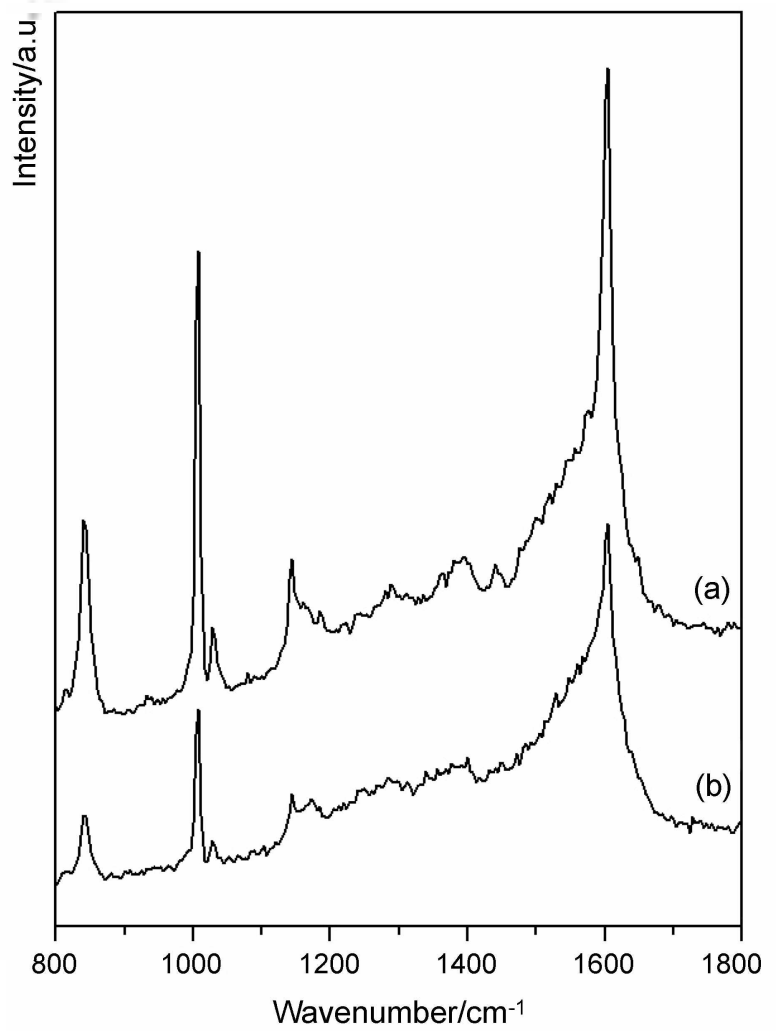

Figure 6. SLRS spectra of $10^{-5} \mathrm{M} \mathrm{BA}$ adsorbed on TRS. (a) obtained from the first scan. (b) obtained from the second scan atter being illuminated with a higher laser excitation porer. 
ylate group with the benzene ring orienting away from the surface normal. The latter group favored a flat or tilted orientation on the sol particle surfaces. Carboxylic acid has two binding sites to metal, one is the oxygen lone pair electrons and the other is the carboxylate $\pi$ electrons. ${ }^{10-22}$ It is interesting to note what kind of orientation and binding sites the BA takes on the Ag-doped sol-gel film. The SERS spectrum of BA obtained using TRS [Figure 5(b)] shows better resolution than that obtained using CRS [Figure 5(a)]. For this reason, we make use of Figure 5 (b) when we discuss the adsorption form, binding site, and orientation of BA. The symmetric stretching and bending vibrations of the carboxylate group are seen in 1392 and $840 \mathrm{~cm}^{-1}$. respectively, and the acid $v(\mathrm{C}=0)$ vibration band at about $1634 \mathrm{~cm}^{-1}$, which is seen in solid state acid [Figure 4], is not observed in the SERS spectrum. This means, undoubtedly, that the BA is adsorbed on 'TRS in the form of benzoate ion.

Referring to the previous SERS studies on carboxylic acids, the $v_{\mathrm{s}}\left(\mathrm{COO}^{-}\right)$mode red-shifted by as much as $13-19$ $\mathrm{cm}^{-1}$ from the value in a free state when the carboxylic acid adsorbed on the surface via the carboxylate $\pi$ electrons. ${ }^{19.2 .7}$ whereas the same mode red-shifted only by $4 \mathrm{~cm}^{-1}$ when it adsorbed on the surface via the oxygen lone pair electrons. ${ }^{18}$ As shown in l-igure 4(b) and l.igure $5(\mathrm{~b})$, and also shown in lable 1 , the $v_{s}\left(\mathrm{COO}^{-}\right)$mode of $\mathrm{BA}$ adsorbed on TRS does not shift, which can be thought that the BA adsorbs on TRS via the oxygen lone pair electrons rather than via the carboxylate $\pi$ electrons. Such a bonding will more favorably

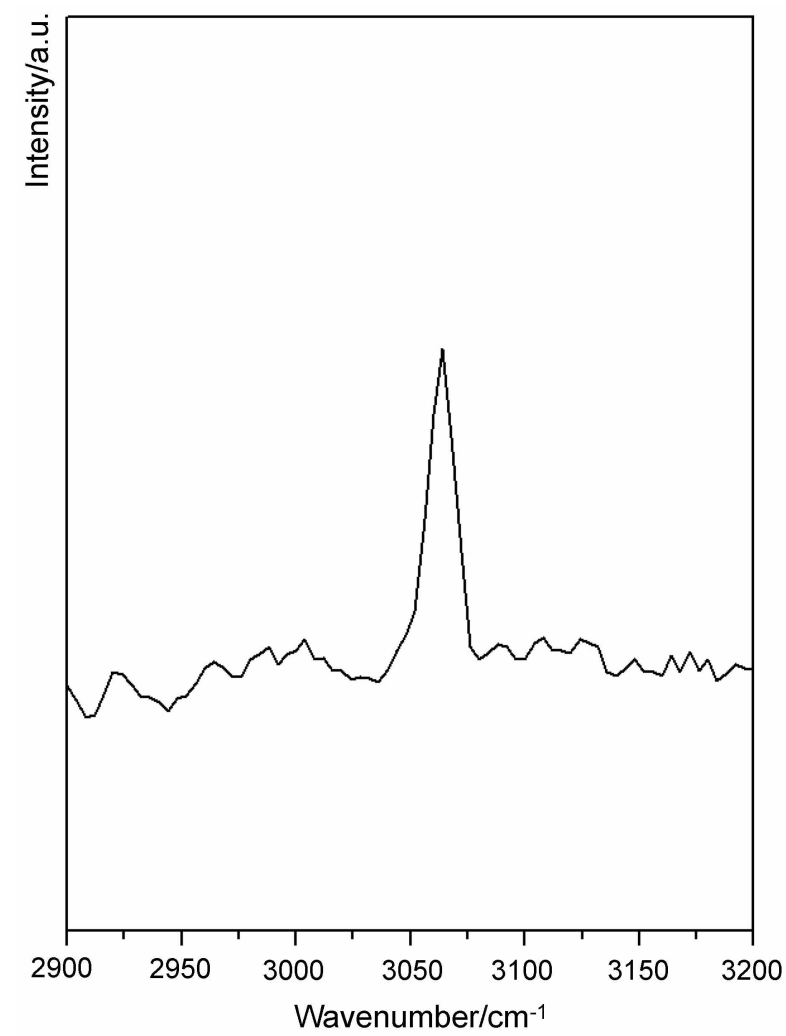

Figure 7. SERS spectrum of 10 " $\mathrm{M}$ BA adsorbed on TRS showing a benzene ring $\mathrm{C}-\mathrm{H}$ stretching band at $3064 \mathrm{~cm}^{-1}$. occur with a perpendicular orientation.

Although the SERS selection rule has not been established precisely, the benzene ring $\mathrm{C}-\mathrm{H}$ stretching vibration was demonstrated to be an obvious probe in the determination of surface orientation of aromatics. Namely, in accordance with the electromagnetic surface selection rule proposed by Creighton $^{24}$ and by Moskovits and Suh, ${ }^{25}$ the $\mathrm{C}-\mathrm{H}$ stretching vibration should be relatively more enhanced when the $\mathrm{C}-\mathrm{H}$ bond is perpendicular to the surface than when it lies parallel to the surface. In this regard. Pagannone et al. ${ }^{1.1}$ suggested that adsorbed benzoate do not lie flat on the surface on the basis of the presence of the $v(\mathrm{C}-\mathrm{H})$ band in its SFRS spectrum. Shown in Figure 7 is the SERS spectrum of BA adsorbed on JRS showing a benzene ring $\mathrm{C}-\mathrm{H}$ stretching band at $3064 \mathrm{~cm}^{-1}$. which tell us that the adsorbed BA on IRS may be more inclined to a perpendicular orientation rather than to a flat one.

\section{Conclusion}

In the present study, we developed new methods to prepare Ag-doped sol-gel filıns for SERS applications and gave an interpretation of the SERS spectra of BA adsorbed on the substrates. Experimental results indicated that the adsorption strength of BA on Ag-doped sol-gel films was different according to the kind of reduction method: BA adsorbed on CRS strongly, whereas it adsorbed on TRS weakly. Knowledge about the form and orientation of adsorbate from the SERS measurements was obtained. The BA molecules adsorbed on TRS as carboxylate ions and oxygen atoms of the carboxylate were bonded to the surface. We suggested that adsorbed BA would favor a perpendicular orientation rather than a flat orientation on JRS, on the basis of the presence of the $v(\mathrm{C}-\mathrm{H})$ band in its SI:RS spectrum.

Acknowledgement. This work was supported by Korea Research Foundation Grant (KRF-2002-KRF-2002-005-C00012 ).

\section{References}

1. Fleischmann. M. 1.: Hendra. P. I.: MeQuillan. A. 1. Chen. Phys. letters 1974. 26. 163.

2. Otto A.: Mrozec, I.: Grabhorn, Il: Akermann, W. J. Phys Condens Matter 1992. f. 114.

3. Nabier. 1.: Chourpa I.: Manlair. M. J. Romem Spectrose: 1994. 25. 13

4. Mood.: R. L.: Vo-Dinh. T.: Fletcher. W. H. Appl. Spectrosc. 1987. 4. 966.

5. (iuhathakurta-Gihosh. V: Arocat R. J. Ptys (hem. 1989. 93. 6128 .

6. IJou. X: Wu. L.: Xu. W: Qin. I..: Wang. C.: Zhang. X.: Shen. I. Colloids and Sarfoces t: Physicochem. Eng. Aspects 2002. 198. 135 .

7. Jung. Y. M.: Lim. J. W.: Kim. E. R.: Lee. H.: Lec. M. S. BuH. Korean ('hem. Soc. 2001, 22, 318.

8. Kang. J. S.: Ilwang. S. Y.: Lee. C. I.: Lee. M. S. Bult. Korean Chent. Soc: 2002. 23. 1604.

9. Special lssue on Raman Scattering for Glasses and Sol-Giel Processes J. Rantam Specthosc. 1996.27. 10. 
10. Akarian. F.: Dunn. B. S.: Zink. J. I. J. Phys. Chem. 1995 99.3892.

II. Meier. M.: Wokaun. A.: Vo-Dinh. T. J. Phvs. Chent 1985.89. 1843.

12. Rowe. J. E.: Shank. C. V: Zwemer. D. A.: Murray C. A. Phus, Rev Lett. 1980. H. 1770 .

13. Varsanyi, G. Assigments for Tibrational Specta of Seven Hondred Benzene Derinatives: Academia Kiado: Budapest. 1974.

14. Alak. A. M.: Vo-Dinh. T. Anal Chem 1989. 61.656.

15. Bello, I. M:Stokes, D. L.: Vo-Dinh. T. Appt. Spectrose. 1989. 13. 1325 .

16. Tsang. J. C.: Demuth, J. E.: Sanda. P. N.: Kirtley, I. R. Chem. Plys. Lett. 1980.71.54.
17. Otto. A. Suface Sci. 1978. 75. 1392.

18. Kwonl. Y. J.: Son1. D. H.: Aht1. S. T.: Kimn. M. S.: Kim. K. J. Phss. Chent 1994. 98. 8481 .

19. Kwon. Y. J.; Lee. S. B.: Kim, M. S.: Kim, K. J. Hol Sintct 1994. 318.25.

20. Upton. T. H. d. Chem. Phys. 1985, 83.5084.

21. Stöhr. T.: Outka. D. A.: Madix. R. T.: Döbler. U. Phus. Rev Lett 1985.57. 1256

22. Sexton. B. A.: Madix. R. J. Sunf. Sci. 1981. 105. 177.

23. Park, H. Lee. S. B.; Kim, K.: Kim. M. S. J. Phys. Chem. $1990,94$. 7576 .

24. Creighton, J. A. Surf. Sci. 1983, 124, 209.

25. Moskorits. M.: Sulh. T. S. J.Am. Chent Soc. 1986. 108.4711. 